\begin{tabular}{|c|l|}
\hline Title & Fast switching of light propagation in a photorefractive crystal via Pockels effect \\
\hline Author(s) & Xie, Ping; Mishima, Teruhito \\
\hline Citation & $\begin{array}{l}\text { Optics Communications, 246(1-3), 29-34 } \\
\text { https://doi.org/10.1016j.optcom.2004.10.071 }\end{array}$ \\
\hline Issue Date & 2005-02-01 \\
\hline Doc URL & http://hdl.handle.net/2115/513 \\
\hline Type & article (author version) \\
\hline File Information & manuscript_revised.pdf \\
\hline
\end{tabular}

Instructions for use 


\title{
Fast switching of light propagation in a photorefractive crystal via Pockels effect
}

\author{
Ping Xie ${ }^{(a)}$ and Teruhito Mishima ${ }^{(b)}$ \\ (a) Institute of Physics, Chinese Academy of Sciences, Beijing 100080, China \\ (b) Division of Electronics for Informatics, Hokkaido University,
}

Sapporo 060-0814, Japan

\begin{abstract}
We experimentally demonstrate fast switching of light propagation and phase conjugate generation in $\mathrm{BaTiO}_{3}: \mathrm{Ce}$ crystal with a high efficiency. The switching mechanism is based on the phase mismatch in photorefractive beam coupling between an incident beam and a fanning beam. The phase mismatch is induced by an externally applied electric field via Pockels effect. In our experiment we obatin a voltage-generator-determined switching time of the order of $10 \mu$ s. The required voltage to reduce the output phase conjugate power by more than $90 \%$ is about only 100 volts for our 3.8 mm-thick crystal.
\end{abstract}

Keywords: Photorefractive effect; Optical switching; Phase conjugation; Pockels effect; Phase mismatch 
Intensive research has been going on in the field of fast and efficient optical switching. The most popular development is the optical switch based on Pockels effect using a $\mathrm{LiNbO}_{3}$ crystal [1]. This type of optical switch can achieve very high speed due to the fast Pockels effect. Another type of switches is the one using absorption by carriers in semiconductor optical modulator, which seems promising in waveguide optics [2]. A switch using the Kerr-nonlinear photonic crystals has also been proposed to achieve efficient optical switch [3].

Photorefractive crystal is an important nonlinear-optical medium. Since the photorefractive effect is capable of efficient beam coupling even at a very low level of powers and has various promising applications in optical phase-conjugate-wave generation, optical processing, optical interconnection and switching, etc., it has attracted a lot of interest of reserchers in the past decades [4,5]. However, a crucial disadvantage of the photorefractive effect is its rather slow temporal response, which is usually larger than the order of seconds. The slow response time greatly limits its applications, particularly in fast optical switching. Recently, we have proposed and experimentally demonstrated that this problem of slow speed of switching can be overcome by applying an electric field to control the efficiency of photorefractive four-wave mixing [6,7].

In this paper, we experimentally study the fast switching of a single beam propagation through a cerium-doped $\mathrm{BaTiO}_{3}$ photorefractive crystal as well as the consequent phase conjugate generation. The switching is achieved by applying an external voltage. The system is rather simple and is very easy to align in practice. The high efficiency of switching with very weak input power and with the applied voltage smaller than 100 volts makes it rather promising in practical applications.

The experimental set-up is schematically shown in Fig.1. An extraordinary beam of wavelength $\lambda=514.5 \mathrm{~nm}$ is incident upon the $\mathrm{BaTiO}_{3}$ : Ce crystal with an angle of $\theta_{0}$ outside 
the crystal, where the angle is defined positive for a clockwise rotation from the surface normal. The doped cerium can enhance the photorefractive effect of $\mathrm{BaTiO}_{3}$, maybe, due to the increased density of acceptor impurity. The dimension of the crystal is $6.1 \mathrm{~mm} \times 4.0 \mathrm{~mm} \times 3.8$ $\mathrm{mm}$ ( $L=3.8 \mathrm{~mm}$ ), with $\mathrm{c}$ axis along the $6.1 \mathrm{~mm}$ edge. To apply an electric field to the crystal a pair of Indium-Tin-Oxide (ITO) transparant electrodes are coated onto the input surface and its opposite surface of the crystal.

At first, we consider the case when there is no external electric field. Owing to the strong photorefractive effect the fanning beam is amplified at the expense of the incident beam power and consequently the counterpropagating phase conjugate beam is generated $[8,9]$. In Fig.2 we show a typical temporal evolution of the power of the transmitted beam at $x=L$ and the output phase conjugate power at $x=0$ for incident angle $\theta_{0}=30^{\circ}$, where the input beam is turned on at $t=0$ and the input power at $x=0$ is $I_{0}=9.1 \mathrm{~mW}$. The steady-state output phase conjugate power is $I_{\mathrm{pc}}=1.24 \mathrm{~mW}$ and the steady-state power of the transmitted beam is $I_{\mathrm{t}}=3.8$ $\mu \mathrm{W}$. (Note that, owing to the poor transparency of the electrodes, the phase conjugate power inside the crystal is in fact larger than that measured here.) It is seen that, due to the slow speed of the photorefractive grating formation, the transmitted power (or the phase conjugate power) takes more than 40 seconds to reach its steady state.

After the power reaches the steady state we apply an electric field $E_{x}$ to the crystal along $x$ axis, as shown in Fig.1. Under the field the refractive indices for the incident and the fanning beams change, due to the Pockels effect, as follows $[6,7]$

$$
\frac{1}{n_{\alpha}^{\prime 2}}=\frac{\sin ^{2} \theta_{\alpha}}{n_{\mathrm{o}}^{2}}+\frac{\cos ^{2} \theta_{\alpha}}{n_{\mathrm{e}}^{2}}-r_{42} \sin 2 \theta_{\alpha} E_{x}, \quad(\alpha=\text { i or } \mathrm{f})
$$

where $\theta_{\mathrm{i}}$ and $\theta_{\mathrm{f}}$ are propagation angles of the incident beam and the fanning beam relative to the $x$ axis, respectively, inside the crystal, $n_{\mathrm{i}}{ }^{\prime}$ and $n_{\mathrm{f}}{ }^{\prime}$ are the refractive indices for the incident beam 
and the fanning beam in the presence of electric field, respectively, while $n_{\mathrm{e}}$ and $n_{\mathrm{o}}$ are the two principal refractive indices of the $\mathrm{BaTiO}_{3}: \mathrm{Ce}$ crystal, and $r_{42}$ is the component of its electro-optic tensor. Here, for simplicity of analysis, we consider the fanning beam with only one propagation direction, $\theta_{\mathrm{f}}$, that corresponds to its Fourier plane-wave compornent of the largest amplified intensity. It should be emphasized that, owing to the fast speed of the Pockels effect (which is usually faster than the order of nanoseconds [10]), the response time of the refractive-index change is much faster than that of the photorefractive grating formation/erasure, which is slower than the order of seconds, as can be seen in Fig.1. During a short period of time, which is justified in the fast optical switching that is the interest of this paper, the built-in photorefractive grating can be considered to be unchanged. On the other hand, the change in refractive index induced by the applied electric field via the Pockels effect, as shown in Eq.(1), can result in the the changes in the wave vectors of the incident and fanning beams inside the crystal. The changes in the wave vectors induce the phase mismatch

$$
\Delta \mathbf{k}=\mathbf{k}_{\mathrm{g}}-\left(\mathbf{k}_{\mathrm{f}}{ }^{\prime}-\mathbf{k}_{\mathrm{i}}{ }^{\prime}\right)
$$

for the diffraction, where $\mathbf{k}_{\mathrm{g}}=\mathbf{k}_{\mathrm{f}}-\mathbf{k}_{\mathrm{i}}$ is the built-in grating. $\mathbf{k}_{\mathrm{i}}{ }^{\prime}\left(\mathbf{k}_{\mathrm{i}}\right)$ and $\mathbf{k}_{\mathrm{f}}{ }^{\prime}\left(\mathbf{k}_{\mathrm{f}}\right)$ are the wave vectors of the incident and the fanning beams in the presence (absence) of the external field $E_{x}$, respectively, the magnitudes of which are approximately derived as

$$
k_{\alpha}^{\prime}=k_{\alpha}\left\{1+\frac{r_{42} \sin 2 \theta_{\alpha} E_{x}}{2\left[\frac{\sin ^{2} \theta_{\alpha}}{n_{\mathrm{o}}^{2}}+\frac{\cos ^{2} \theta_{\alpha}}{n_{\mathrm{e}}^{2}}\right]}\right\} \quad(\alpha=\mathrm{i} \text { or } \mathrm{f})
$$

from Eq.(1).

From Eqs. (2) and (3) it is easy to see that, for the large value of $r_{42}$ in $\mathrm{BaTiO}_{3}: \mathrm{Ce}$ crystal, when the difference between $\theta_{\mathrm{f}}$ and $\theta_{\mathrm{i}}$ is large a mediate external field can induce a phase mismatch to reduce diffraction to a great extent. Thus, efficient switching of the 
transmitted beam and consequent switching of the phase conjugate beam can be achieved. The switching time is the same as the response time of the Pockels effect of the crystal. The switching time cannot be limited by the time of the grating formation/erasure.

In Figs. 3 and 4 we show some examples of our measured temporal evolution of the power of the transmitted beam and that of the phase conjugate beam with an external ac voltage. The experimental conditions are the same as those in Fig.2, i.e., with the same incident angle $\theta_{0}=30^{\circ}$ and input power $I_{0}=9.1 \mathrm{~mW}$. Figure 3 plots the power of the transmitted beam and Fig. 4 plots the output power of the phase conjugate beam. In Fig. 3(a), at $t<0.525 \mathrm{~s}$ the voltage is off. At $t \approx 0.526 \mathrm{~s}$ we turn on a $50 \mathrm{~Hz}$ square-form voltage, the high and the low values of which are 0 and -114 volts, respectively. The voltage of -114 volts corresponds to the electric field of -30 volts $/ \mathrm{mm}$. In Fig. 3(b), at $t \approx 0.842 \mathrm{~s}$ we turn on the similar voltage as in Fig. 3(a) but with the low and the high values being 0 and +114 volts, respectively. From both Figs. 3(a) and 3(b) we see that, since the grating has been previously formed under no external field, the transmitted power $I_{\mathrm{t}}$ increases to a larger value when the voltage changes from 0 to the other values.

From Fig. 3(c), which is the elongation of Fig.3(b) along the time axis, we see that the switching time of the transmitted power is about $30 \mu \mathrm{s}$, which is exactly the same as the switching time of the applied voltage that is determined by our voltage generator, not by the time of the grating formation/erasure. This is because the switching time of the voltage generator is slower than the response time of the Pockels effect of our crystal, which is estimated to be the order of $0.1 \mathrm{~ns}[10]$, and, therefore, the switching time of the transmitted power is only determined by the voltage generator in our experiment.

Similarly, Fig. 4(a) shows the temporal evolution of the phase-conjugate output power with a $50-\mathrm{Hz}$ square-form voltage. The high and the low values of the voltage are 0 and -114 
volts, respectively. Figure 4(b) is with the similar voltage, but with the low and the high values being 0 and +114 volts, respectively. Contrary to the transmitted power as shown in Fig. 3, the phase conjugate power decreases from a high value $I_{\mathrm{pc}}(0)$ to a very low value $I_{\mathrm{pc}}(V)$ simultaneously as the voltage changes from 0 to \pm 114 volts as shown in Figs. 4(a) and 4(b). The power increases from a very low value $I_{\mathrm{pc}}(V)$ to a high value $I_{\mathrm{pc}}(0)$ simultaneously as the voltage changes from \pm 114 to 0 volts, also as shown in Figs. 4(a) and 4(b).

To see the efficiency of the switching, we show $I_{\mathrm{t}}(V) / I_{\mathrm{t}}(0)$ and $I_{\mathrm{pc}}(V) / I_{\mathrm{pc}}(0)$ as a function of the applied voltage in Figs. 5(a) and 5(b), respectively, for various incident angles $\theta_{0}$. We use the same input power $I_{0}=9.1 \mathrm{~mW}$ for all $\theta_{0}$ in Fig. 5 and the measured steady-state output powers of the phase conjugate beam without applied field are $I_{\mathrm{pc}}(0)=1.24 \mathrm{~mW}$ for $\theta_{0}=30^{\circ}, 195 \mu \mathrm{W}$ for $\theta_{0}=2.5^{\circ}$, and $61 \mu \mathrm{W}$ for $\theta_{0}=-30^{\circ}$. From both Figs. 5(a) and 5(b) we note that the efficiency of switching is nearly symmetric for positive and negative voltages and it increases with the increase of the magnitude of the voltage, as can be noted from Eqs. (2) and (3). From Fig. 5(b) we see that the switching is more effective for a smaller $\theta_{0}$ than for a larger $\theta_{0}$. This can be understood as follows: As $\theta_{0}$ decreases, the difference between angles $\theta_{\mathrm{f}}$ and $\theta_{\mathrm{i}}$ increases since in $\mathrm{BaTiO}_{3}:$ Ce crystal the energy is mostly transferred to the plane-wave component of the fanning beam with a smaller angle relative to c axis [9]. From Eqs. (2) and (3) it is noted that the increase of this difference leads to the increase of the phase mismatch, which results in more effective switching. From Fig. 5(b) it is seen that, to reduce the phase conjugate power by $90 \%$, the applied voltage required is only about 70 volts for $\theta_{0}=-30^{\circ}$, which can be easily implemented in practice.

In conclusion, we have experimentally demonstrated fast switching of the light propagation as well as of the phase conjugate generation in a $\mathrm{BaTiO}_{3}: \mathrm{Ce}$ crystal with a high 
efficiency. The switching mechanism is based on the phase mismatch in the photorefractive beam coupling between the incident beam and the fanning beam. The phase mismatch is induced by an externally applied electric field via the Pockels effect, the response time of which is of the order of $0.1 \mathrm{~ns}$. In our experiment we obatin a voltage-generator-determined switching time of about $30 \mu \mathrm{s}$. The required voltage to reduce the output phase conjugate power by more than $90 \%$ is about only 100 volts. The high efficiency of the switch with very weak input power and with the applied voltage of only 100 volts makes it rather promising in future practical applications.

\section{Acknowledgments}

This work was supported by a Grant-in-Aid for Scientific Research (B), the Ministy of Education, Culture, Sports, Science and Techonology, Japan. 


\section{References}

[1] A. Yariv, Optical Electronics, $4^{\text {th }}$ ed., Saunders College Publishing, Philadelphia, 1991 (Chapters 9, and 18).

[2] I. Ogawa, F. Ebisawa, F. Hanawa, T. Hashimoto, M. Yanagisawa, K. Shuto, T. Ohyama, Y. Yamada, Y. Akahori, A. Himeno, K. Kato, N. Yoshimoto, Y. Tohmori, Electronics Lett., 34, $361,1998$.

[3] P. Xie, Z.-Q. Zhang, J. Appl. Phys. 95, 1630, 2004.

[4] P. Yeh, Introduction to Photorefractive Nonlinear Optics, Wiley, New York, 1993.

[5] See, for example, P. Günter and J.-P. Huignard, eds., Photorefractive Materials and Their Applications I, II, Vols. 61 and 62 of Topics in Applied Physics, Springer-Verlag, Berlin $(1988,1989)$.

[6] I.A. Taj, P. Xie, T. Mishima, Opt. Commun. 187, 7 (2001).

[7] T. Mishima, P. Xie, K. Koyanagi, I. A. Taj, Opt. Commun. 225, 211 (2003).

[8] P. Xie, J.H. Dai, P.Y. Wang, H.J. Zhang, Appl. Phys. Lett. 69, 4005 (1996).

[9] P. Xie, J.H. Dai, P.Y. Wang, H.J. Zhang, Phys. Rev. A 55, 3092 (1997).

[10] A. Yariv, Quantum Electronics, $2^{\text {nd }}$ ed., John Wiley \& Sons, Inc., 1975 (Ch. 14). 


\section{Figure Captions}

Fig. 1. Schematic diagram of the experimental set up. BS represents a beam splitter, $I_{0}$ is the input power, $I_{\mathrm{t}}$ is the power of the transmitted beam, and $I_{\mathrm{pc}}$ is the output power of the phase conjugate beam. $\theta_{0}$ is the incident angle outside the crystal, $\theta_{\mathrm{i}}$ and $\theta_{\mathrm{f}}$ are propagation angle of the incident beam and fanning beam relative to the $x$ axis, respectively, inside the crystal.

Fig. 2. Temporal evolution of the transmitted power $I_{\mathrm{t}}$ and of the output phase conjugate power $I_{\mathrm{pc}}$ under no applied electric field. The input power $I_{0}$ is turned on at $t=0$.

Fig. 3. Temporal evolution of the transmitted power $I_{\mathrm{t}}$ with an ac square-form external voltage applied to the crystal after the system reaches the steady state. (c) is the elongation of (b) along the time axis.

Fig. 4. Temporal evolution of the output phase conjugate power $I_{\mathrm{pc}}$ with an ac square-form external voltage applied to the crystal after the system reaches its steady state.

Fig. 5. $I_{\mathrm{t}}(V) / I_{\mathrm{t}}(0)$ (a) and $I_{\mathrm{pc}}(V) / I_{\mathrm{pc}}(0)$ (b) as a function of the applied voltage $V$ for incident angle $\theta_{0}=30^{\circ}$ (lines with filled rhombuses), $\theta_{0}=2.5^{\circ} \quad$ (lines with unfilled squares) and $\theta_{0}=-30^{\circ}$ (lines with filled triangles). 
Fig.1 P. Xie, et al.

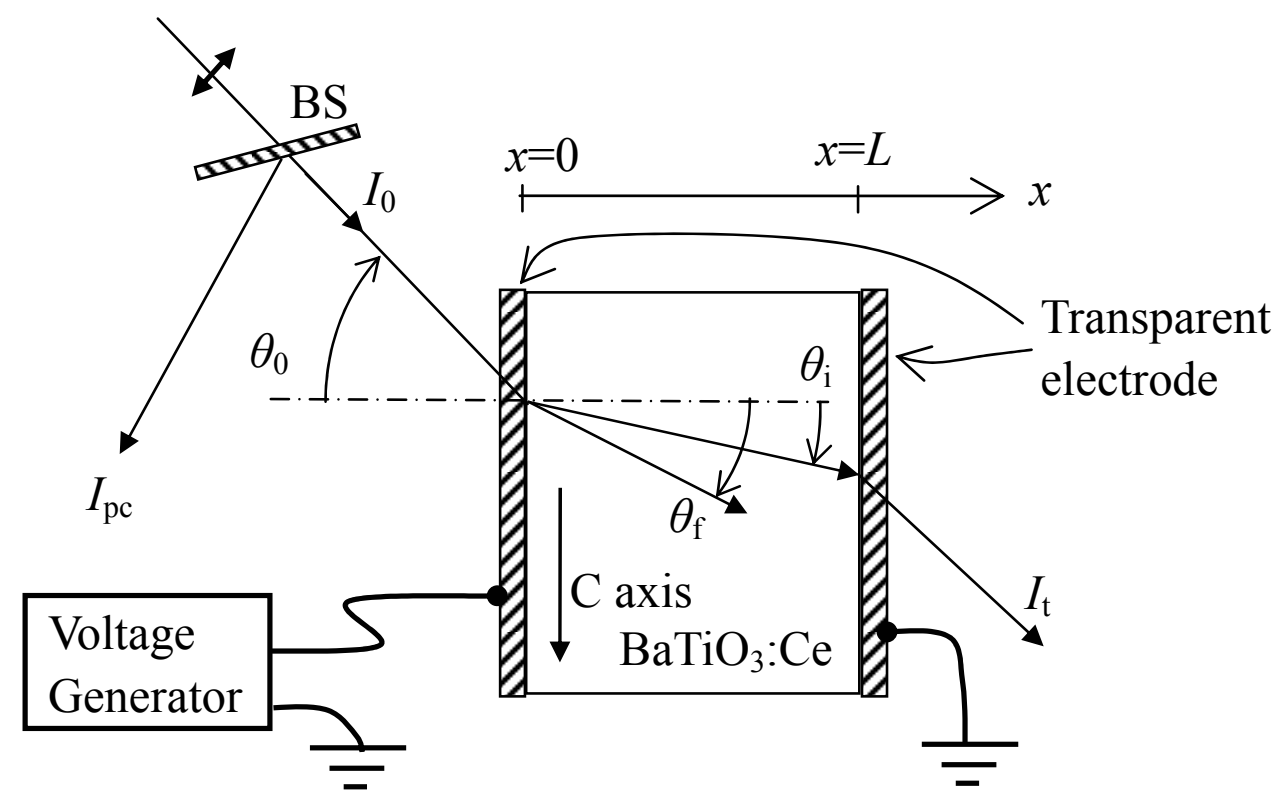


Fig.2 P. Xie, et al.

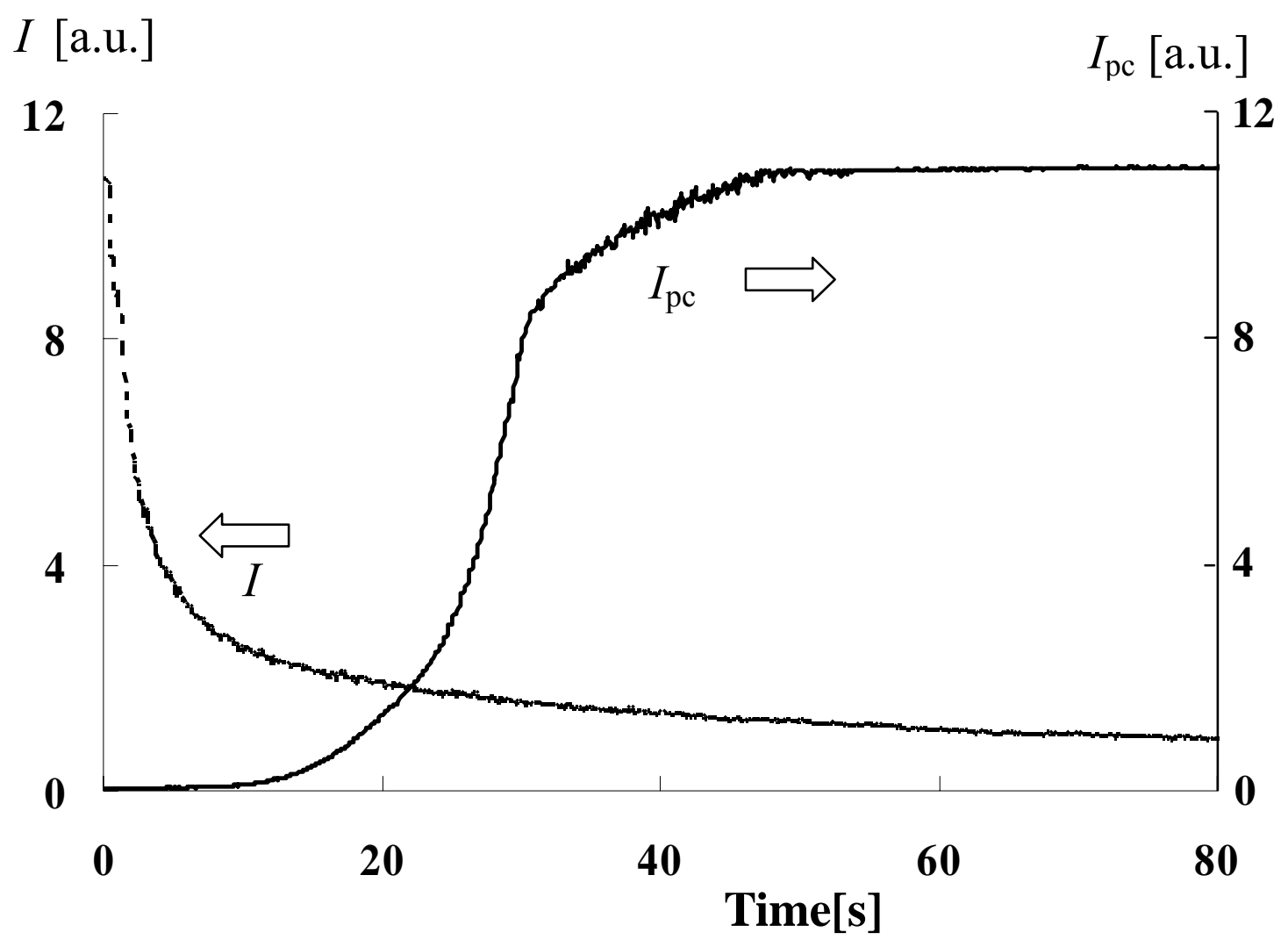


Fig.3(a) P. Xie, et al.

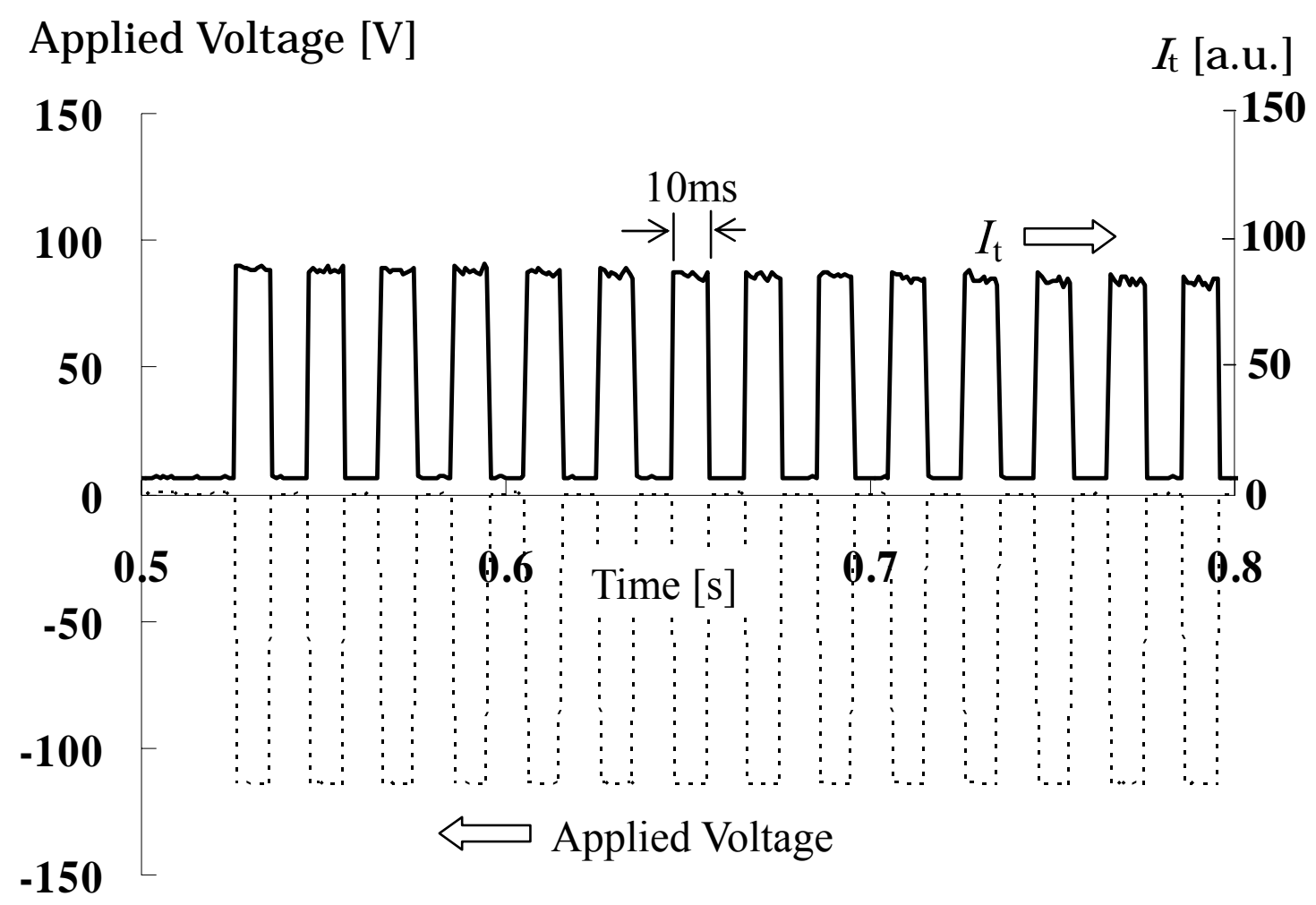


Fig3(b) P. Xie et al.

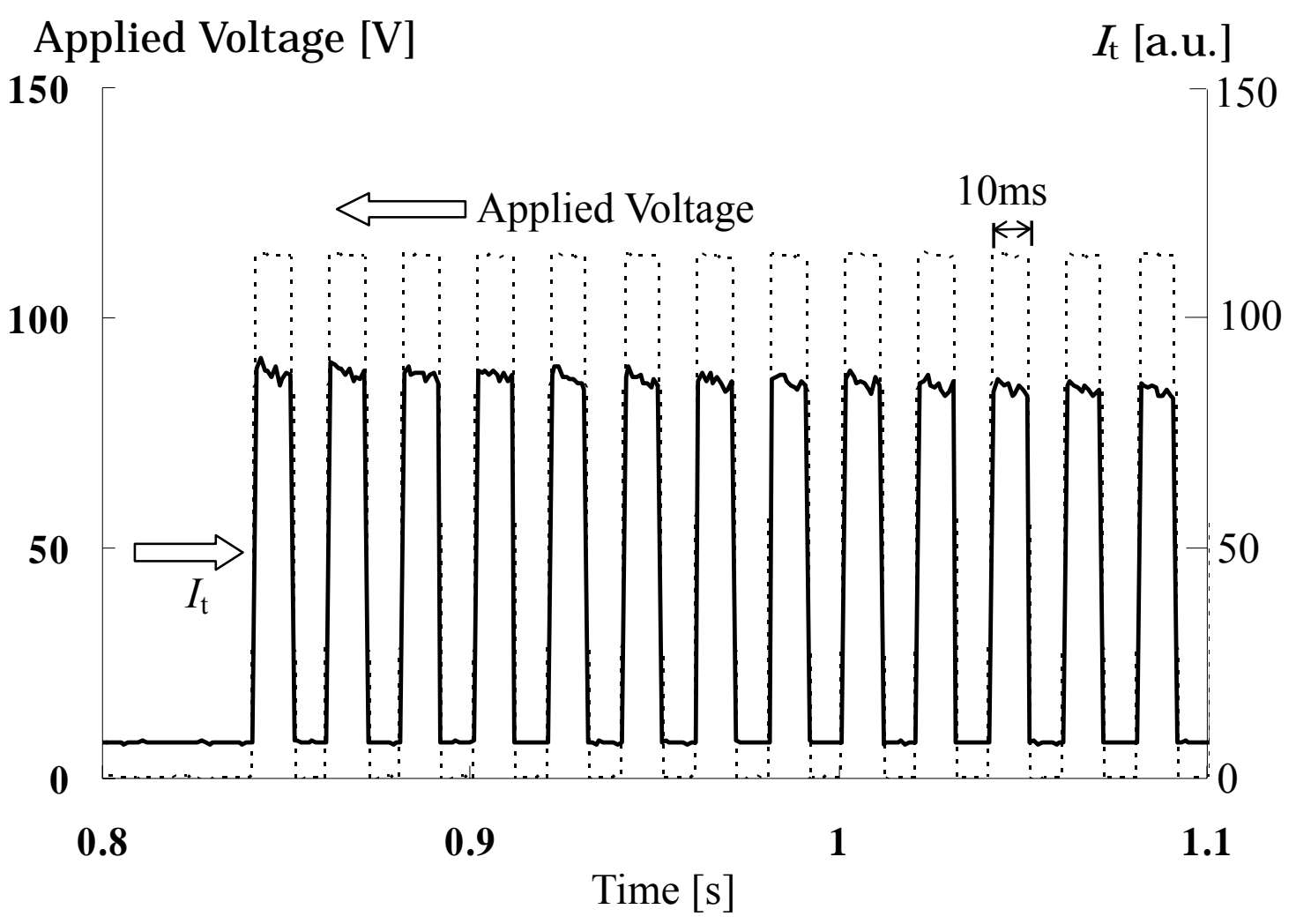


Fig.3 (c) P. Xie, et al.

Applied Voltage [V]

$I_{\mathrm{t}}$ [a.u.]

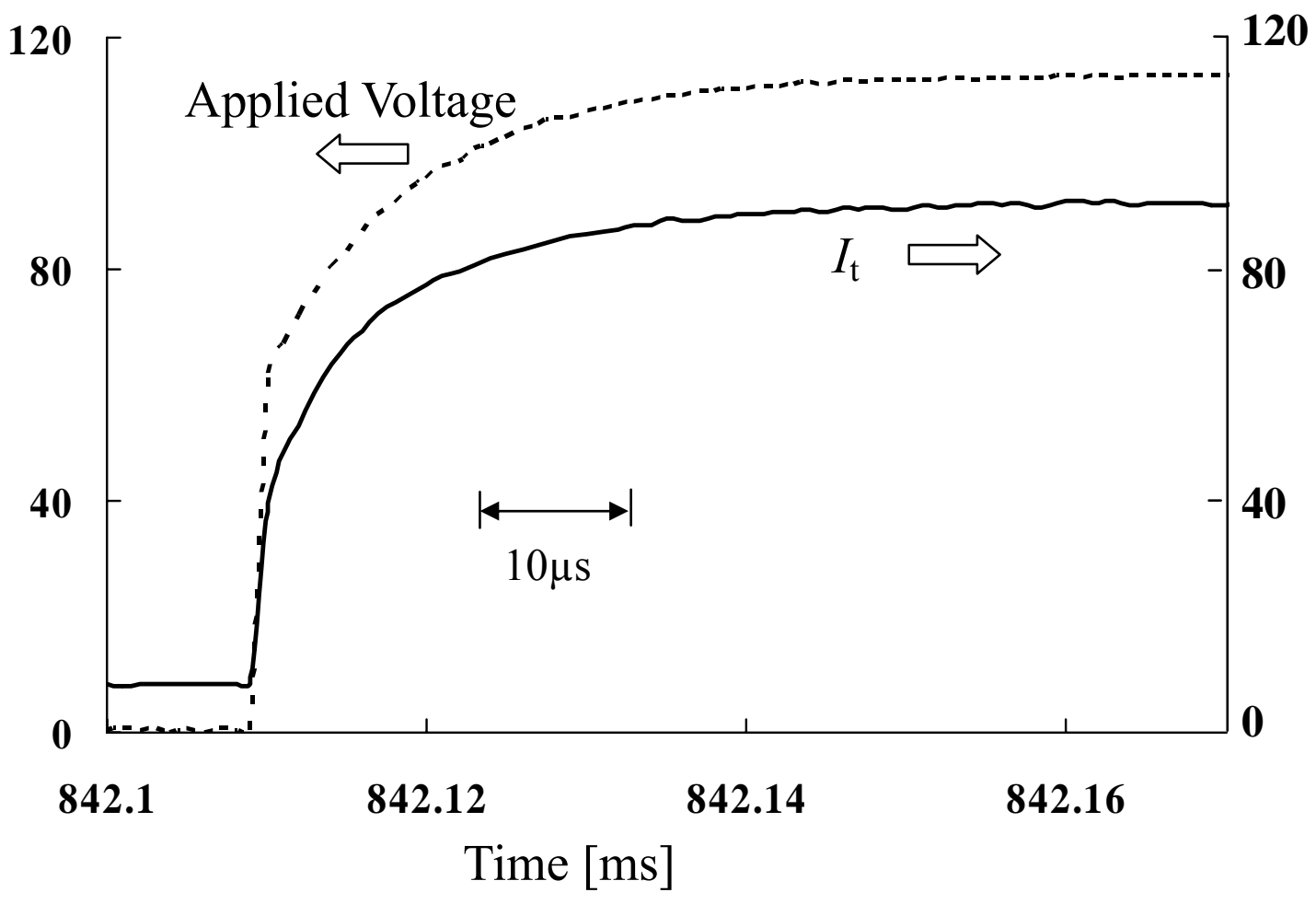


Fig.4(a) P. Xie, et al.

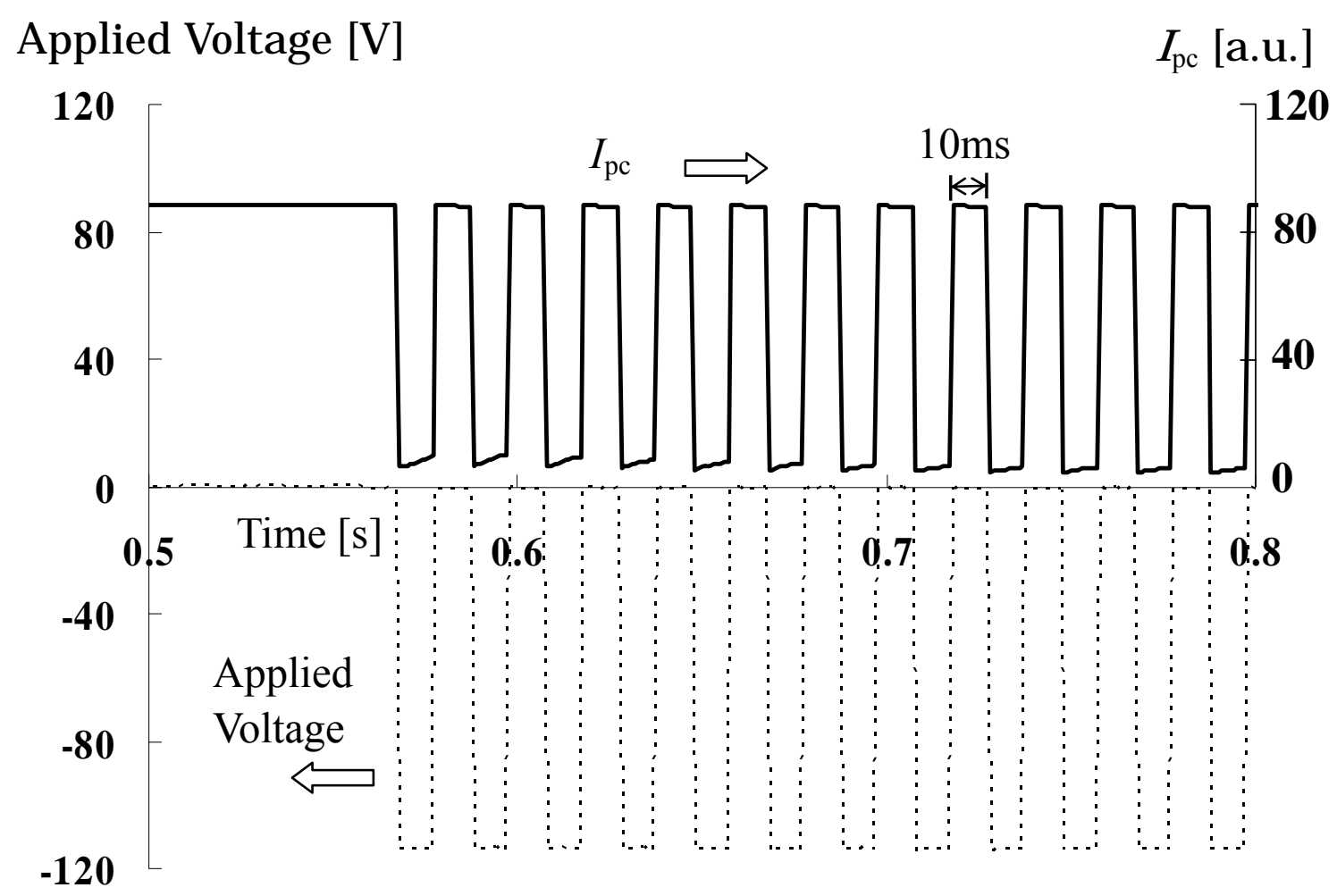


Fig.4(b) P. Xie, et al.

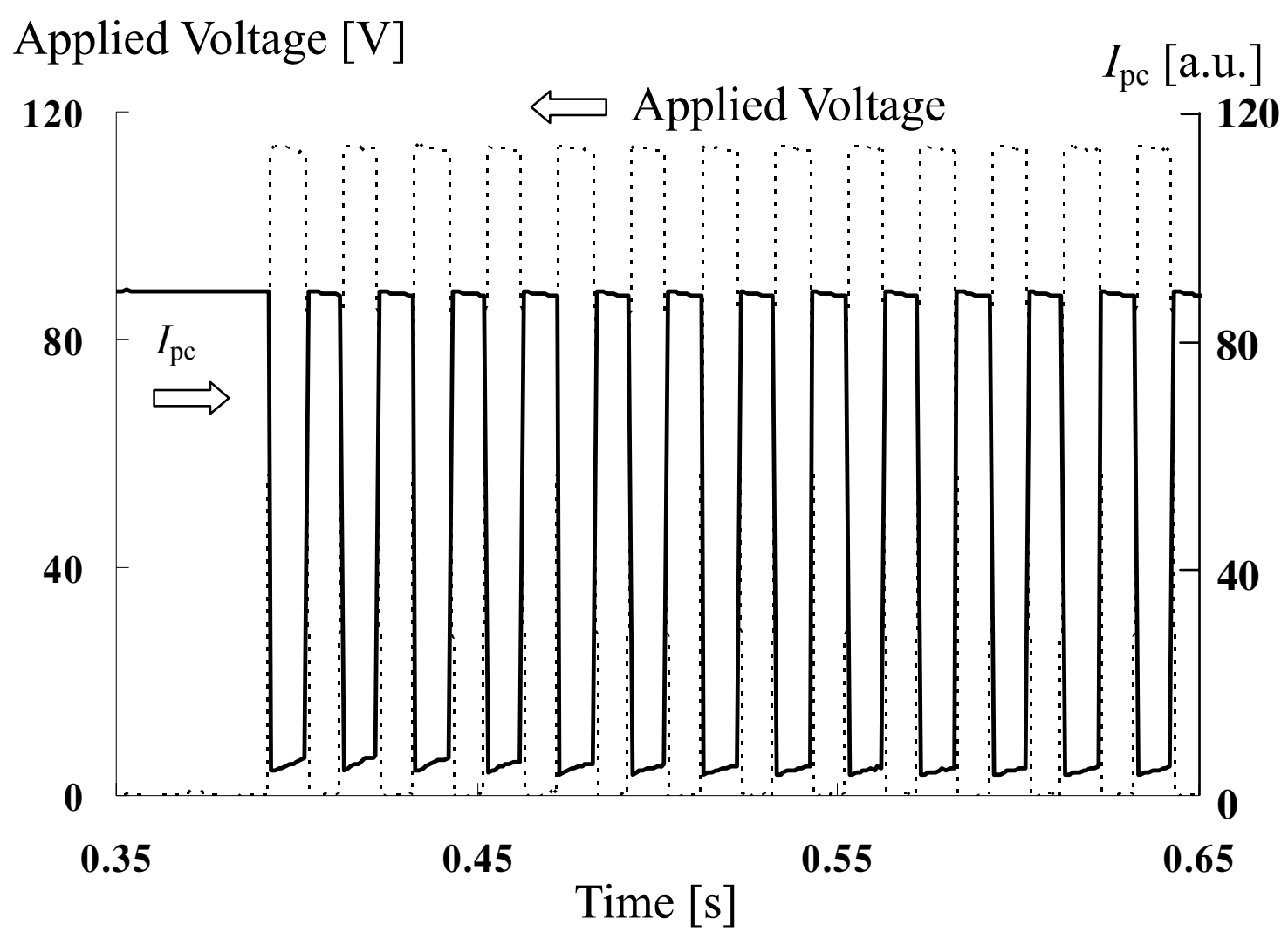


Fig.5(a) P. Xie, et al.

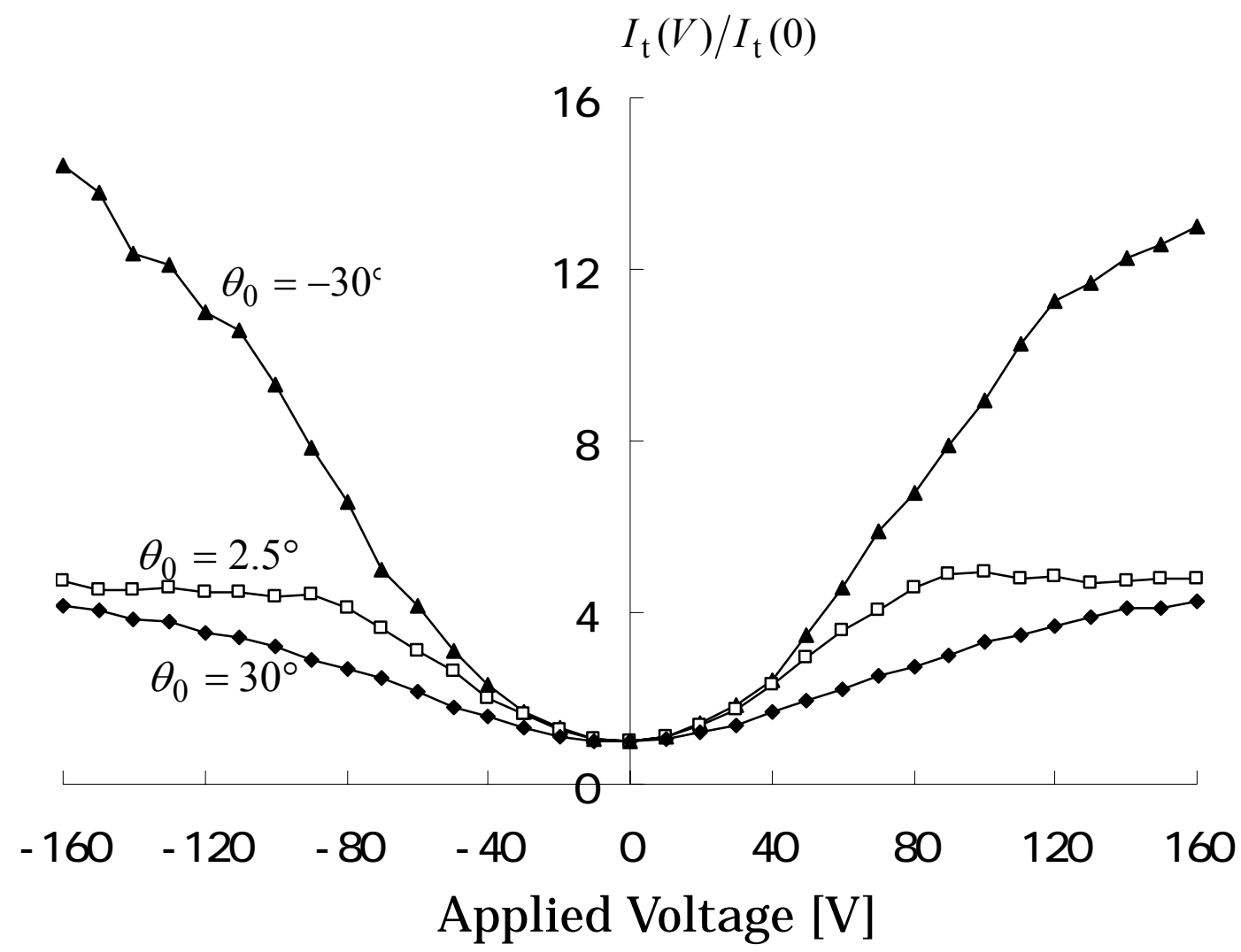


Fig5(b) P. Xie, et al.

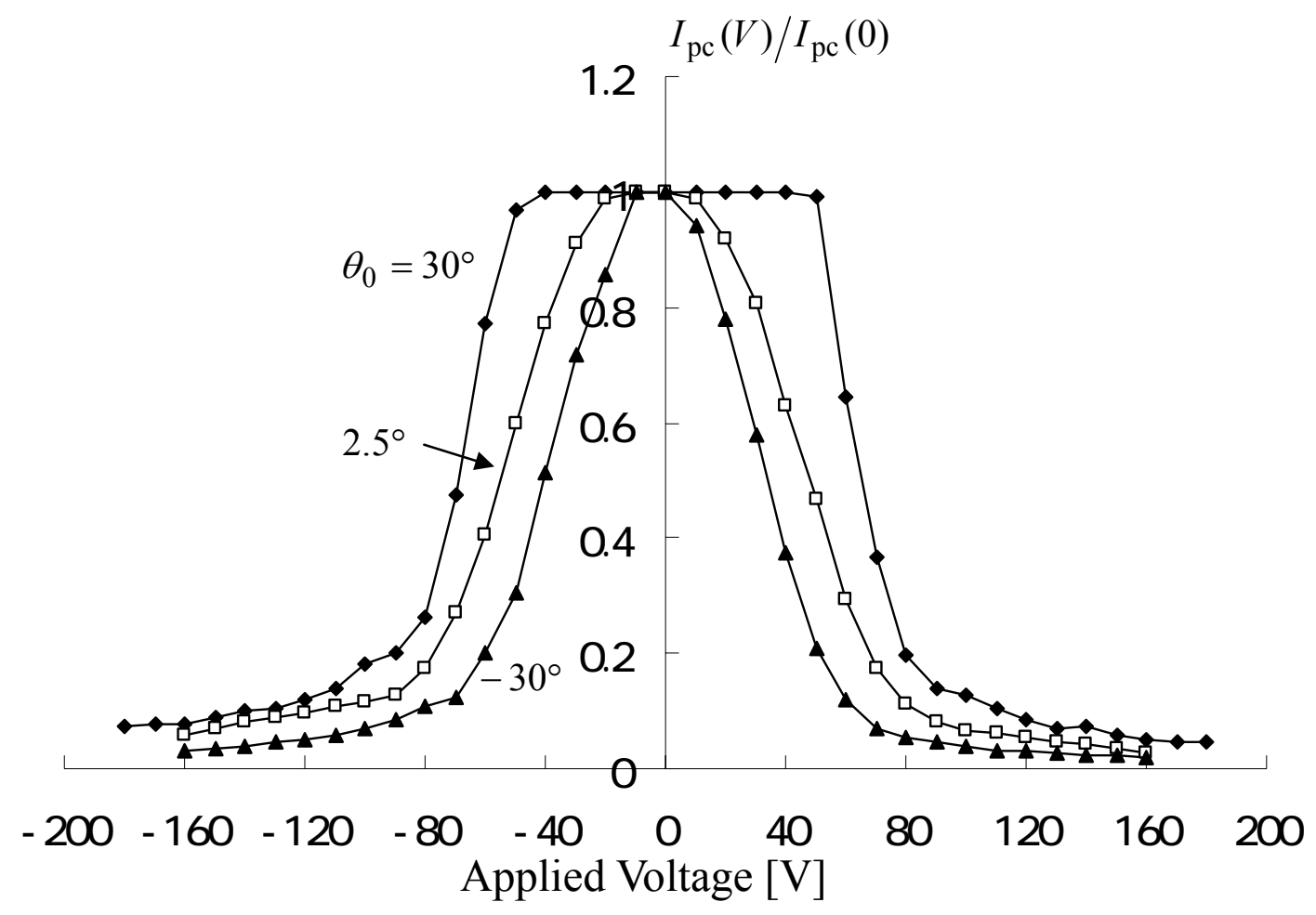

